\title{
The greatest prime factor of the integers in an interval
}

\author{
by \\ HONG-QUAN LiU (Harbin)
}

Introduction. Let $P(x)$ denote the greatest prime factor of

$$
\prod_{x \leq n \leq x+x^{1 / 2}} n,
$$

where $x$ is a large positive number. To estimate the lower bound of $P(x)$ is one of the "greatest prime factor" problems to which the ChebyshevHooley's machinery can be applied. Chronologically Ramachandra [11], [12], Graham [4], Baker [1], and Jia [7] have contributed to the topic. In particular, Baker [1] proved

$$
P(x)>x^{0.7} .
$$

Jia [7] improved the above result. But as [7] contained a substantial mistake concerning a multiple exponential sum (which was already picked out by the present author in 1987), the announced estimate $P(x)>x^{0.71}$ was actually not attainable there.

We prove the following better estimate.

\section{THEOREM.}

$$
P(x)>x^{0.723} .
$$

We begin with

$$
x^{1 / 2} \ln x+O\left(x^{1 / 2}\right)=\sum_{p \leq P(x)} N(p) \ln p, \quad p \text { primes },
$$

where

$$
N(p)=\sum_{x \leq p n \leq x+x^{1 / 2}} 1 .
$$

Thus to prove the Theorem we need to show

$$
\sum:=\sum_{p \leq x^{0.723}} N(p) \ln p<(1-\varepsilon) x^{1 / 2} \ln x, \quad \varepsilon>0 .
$$


For a suitable number $\sigma, 1 / 2<\sigma<0.723$, we write

$$
\sum_{1}=\sum_{p \leq x^{\sigma}} N(p) \ln p, \quad \sum_{2}=\sum_{x^{\sigma} \leq p \leq x^{0.723}} N(p) \ln p .
$$

We need to establish an asymptotic formula for $\sum_{1}$ with $\sigma$ as large as possible, and to estimate $\sum_{2}$ via the sieve.

The proof, as usual, depends mainly on treating some exponential sums, in particular we can prove an asymptotic formula for $\sum_{1}$ with $\sigma=0.6-$ $\varepsilon$. We also make an innovation in the sieve part. To save space we do not analyze the method term by term; the interested reader can find the advantages by himself.

Notations. $e(\xi)=\exp (2 \pi i \xi) .[\xi]$ is the integer part of $\xi$, and $\{\xi\}=$ $\xi-[\xi],\|\xi\|=\min (\{\xi\}, 1-\{\xi\})$.

$$
\sum_{\left(x_{1}, x_{2}, \ldots\right) \in D}
$$

means summation over all lattice points $\left(x_{1}, x_{2}, \ldots\right)$ inside a domain $D$. $f(x, y) \widetilde{\Delta} g(x, y)$ means that

$$
f_{x^{i} y^{j}}(x, y)=g_{x^{i} y^{j}}(x, y)+O\left(\Delta g_{x^{i} y^{j}}(x, y)\right)
$$

whenever both sides make sense. $x \sim X$ means $X \leq x<2 X, x \cong X$ means $C_{1} X \leq x \leq C_{2} X$ for some constants $C_{1}$ and $C_{2}$. The meaning of $x=O(X)$ or $x \ll X$ is as usual. $\varepsilon$, of course, is a sufficiently small number.

1. An asymptotic formula. In this section, we prove

Proposition 1. For $\sigma=0.6-\varepsilon$, we have

$$
\sum_{1}=(0.6-\varepsilon) x^{1 / 2} \ln x+O\left(x^{1 / 2}\right) .
$$

As

$$
N(n)=\frac{x^{1 / 2}}{n}+\psi\left(\frac{x+x^{1 / 2}}{n}\right)-\psi\left(\frac{x}{n}\right), \quad \psi(\xi)=\frac{1}{2}-\{\xi\},
$$

in view of the Prime Number Theorem, to prove (2) it remains to show that (with $\Lambda(n)$ being the von Mangoldt function)

$$
\sum_{x^{1 / 2} \leq n \leq x^{0.6-\varepsilon}} \Lambda(n)\left(\psi\left(\frac{x+x^{1 / 2}}{n}\right)-\psi\left(\frac{x}{n}\right)\right) \ll x^{1 / 2} .
$$

We split the range $x^{1 / 2} \leq n \leq x^{0.6-\varepsilon}$ into ranges of the form $v \leq n \leq v^{\prime}$, $v^{\prime} \leq 2 v$. Then we need to show that

$$
\text { (*) } \sum_{v \leq n \leq v^{\prime}} \Lambda(n) f(n) \ll x^{1 / 2}(\ln x)^{-1}, \quad f(n)=\psi\left(\frac{x+x^{1 / 2}}{n}\right)-\psi\left(\frac{x}{n}\right) .
$$


In view of [1], $(*)$ holds for $x^{1 / 2} \ll v \ll x^{13 / 22-\varepsilon}$, thus we only need to consider the range $x^{0.59} \leq v \leq x^{0.6-\varepsilon}$. We appeal to the following decomposition.

LEMmA 1.1. Let $3 \leq u<w<z \leq 2 v$, suppose that $z-1 / 2$ is an integer, and $z \geq 4 u^{2}, v \geq 32 z^{2} u, w^{3} \geq 64 v$. Set

$$
\begin{gathered}
U=\max _{B} \sum_{m=1}^{\infty} d_{3}(m)\left|\sum_{\substack{z<n<B \\
v \leq m n \leq v^{\prime}}} f(m n)\right|, \\
W=\sup _{g} \sum_{m=1}^{\infty} d_{4}(m)\left|\sum_{\substack{u \leq n \leq w \\
v \leq m n \leq v^{\prime}}} g(n) f(m n)\right|,
\end{gathered}
$$

where the supremum is taken over all arithmetic functions $g(n)$ such that $|g(n)| \leq d_{3}(n)$. Then

$$
U \ll x^{1 / 2}(\ln x)^{-10} \quad \text { and } \quad W \ll x^{1 / 2}(\ln x)^{-10}
$$

imply the estimate

$$
\sum_{v \leq n \leq v^{\prime}} \Lambda(n) f(n) \ll x^{1 / 2}(\ln x)^{-1} .
$$

Proof. See [5].

Choose

$$
u=v x^{-1 / 2+15 \eta}, \quad w=x^{2} v^{-3} x^{-10 \eta}, \quad z=\left[x^{1 / 4-10 \eta}\right]+\frac{1}{2}
$$

in Lemma 1.1, where $\eta=\varepsilon^{2}, x^{0.59} \ll v \ll x^{0.6-\varepsilon}$. Then it is easy to verify that Lemma 1.1 is applicable. As in [1], by a reduction using the Fourier expansion of the function $\psi(\xi)$, in order to show (3) it suffices to demonstrate, with $H=v x^{-1 / 2+\eta}$, the following estimates:

$$
\sum_{0<h \leq H}\left|\sum_{m \sim M} a(m) \sum_{n \sim N} e\left(\frac{h x^{\prime}}{m n}\right)\right| \ll v x^{-\eta}
$$

whenever $v \cong M N, N \gg z,|a(m)| \leq x^{\eta}, x \cong x^{\prime}$; and

$$
\sum_{0<h \leq H}\left|\sum_{m \sim M} a(m) \sum_{n \sim N} b(n) e\left(\frac{h x^{\prime}}{m n}\right)\right| \ll v x^{-\eta}
$$

whenever $v \cong M N, u \ll N \ll w,|a(m)|,|b(n)| \leq x^{\eta}, x \cong x^{\prime}$.

LEMMA 1.2. (5) holds for $v$ in the (larger) range $v \leq x^{5 / 8-\varepsilon}$.

Proof. This is Lemma 9 of [1].

Now it remains to show

LEMMA 1. (4) holds. 
We cite three lemmata of basic importance.

LEMMA 1.3. Let I be a subinterval of $[X, 2 X], Q$ be a positive number, and $z_{n}(X \leq n \leq 2 X)$ be complex numbers. Then

$$
\left|\sum_{n \in I} z_{n}\right|^{2} \leq\left(1+X Q^{-1}\right) \sum_{|q| \leq Q}\left(1-|q| Q^{-1}\right) \sum_{n, n+q \in I} \bar{z}_{n} z_{n+q} .
$$

Proof. This is Weyl's inequality. Cf. [1].

LEMMA 1.4. Let $f(x)$ and $g(x)$ be algebraic functions in the interval $[a, b]$, and

$$
\begin{gathered}
\left|f^{\prime \prime}(x)\right| \cong R^{-1}, \quad\left|f^{\prime \prime \prime}(x)\right| \ll(R U)^{-1} \\
|g(x)| \leq H, \quad\left|g^{\prime}(x)\right| \ll H U_{1}^{-1}, \quad U, U_{1} \geq 1 .
\end{gathered}
$$

Then

$$
\begin{aligned}
\sum_{a \leq n \leq b} g(n) e(f(n))= & \sum_{\alpha \leq u \leq \beta} b_{u} \frac{g(n(u))}{\sqrt{f^{\prime \prime}(n(u))}} e(f(n(u))-u n(u)+1 / 8) \\
& +O\left(H \ln (\beta-\alpha+2)+H(b-a+R)\left(U^{-1}+U_{1}^{-1}\right)\right) \\
& +O\left(H \min \left(R^{1 / 2}, \max (1 /\langle\alpha\rangle, 1 /\langle\beta\rangle)\right)\right),
\end{aligned}
$$

where $[\alpha, \beta]$ is the image of $[a, b]$ under the mapping $y=f^{\prime}(x), n(u)$ is determined by the equation $f^{\prime}(n(u))=u, b_{u}=1 / 2$ or 1 according as $u$ is one of $\alpha$ and $\beta$ or not, and $\langle x\rangle$ is defined as follows:

$$
\langle x\rangle= \begin{cases}\|x\| & \text { if } x \text { is not an integer }, \\ \beta-\alpha & \text { if } x \text { is an integer. }\end{cases}
$$

Moreover, $\sqrt{f^{\prime \prime}}>0$ if $f^{\prime \prime}>0, \sqrt{f^{\prime \prime}}=i \sqrt{\left|f^{\prime \prime}\right|}$ if $f^{\prime \prime}<0$.

Proof. This is Theorem 2.2 of S. H. Min [10].

LEMmA 1.5. Let $f(x, y)$ be an algebraic function in the rectangle $D_{0}=$ $\{(x, y) \mid x \sim X, y \sim Y\}, f(x, y) \widetilde{\Delta} A x^{\alpha} y^{\beta}$ hold throughout $D_{0}$, and $D$ be a subdomain of $D_{0}$ bounded by $O(1)$ algebraic curves. Suppose that $X \geq Y$, $N=X Y, A>0, F=A X^{\alpha} Y^{\beta}, \alpha \beta(\alpha+\beta-1)(\alpha+\beta-2) \neq 0,0<\Delta<\varepsilon_{0}$, where $\varepsilon_{0}$ is a small number depending at most on $\alpha$ and $\beta$. Then

$$
\begin{aligned}
& \sum_{(x, y) \in D} e(f(x, y)) \ll_{\varepsilon, \alpha, \beta}\left(\sqrt[6]{F^{2} N^{3}}+N^{5 / 6}+\sqrt[10]{\Delta^{4} Y^{4} F^{2} N^{5}}\right. \\
& \\
&+\sqrt[8]{F^{-1} X^{-1} N^{8}}+N F^{-1 / 4} \\
&\left.+\sqrt[4]{\Delta X^{-1} N^{4}}+N Y^{-1 / 2}\right)(N F)^{\eta}
\end{aligned}
$$

Proof. This is Lemma 1 of [9]. 
We also need the following auxiliary lemma.

Lemma 1.6. Let $M \leq N<N_{1} \leq M_{1}$, and $a_{n}\left(M \leq n \leq M_{1}\right)$ be complex numbers. Then

$$
\left|\sum_{N<n \leq N_{1}} a_{n}\right| \leq \int_{-\infty}^{\infty} K(\theta)\left|\sum_{M<m \leq M_{1}} a_{m} e(\theta m)\right| d \theta
$$

with $K(\theta)=\min \left(M_{1}-M+1,(\pi|\theta|)^{-1},(\pi \theta)^{-2}\right)$, and

$$
\int_{-\infty}^{\infty} K(\theta) d \theta \leq 3 \ln \left(2+M_{1}-M\right)
$$

Proof. This is Lemma 2.2 of [2].

Proof of Lemma 1. For notational simplicity, we let $x^{\prime}=x$ in (4). For $1 \leq h \leq H$, let

$$
S(h)=\sum_{m \sim M} a(m) \sum_{n \sim N} e\left(\frac{h x}{m n}\right) .
$$

By Lemma 1.4, after a partial summation, we obtain

(6) $x^{-\eta} S(h) \ll\left(\frac{v N^{2}}{h x}\right)^{1 / 2} \sum_{m \sim M}\left|\sum_{u \in I(m)} e(F(u, m))\right|+\left(\frac{v^{3}}{h x}\right)^{1 / 2}+\frac{v^{2}}{h x}+M$,

where $I(m)$ is a subinterval of $\left[C_{1} U, C_{2} U\right], U=h x v^{-1} N^{-1}, F(u, m)=$ $C_{3}\left(h_{x u m^{-1}}\right)^{1 / 2}$ (for $i \geq 1, C_{i}$ denotes a constant). By Lemma 1.6 we get

$$
x^{-\eta} \sum_{m \sim M}\left|\sum_{u \in I(m)} e(F(u, m))\right| \ll \sum_{m \sim M}\left|\sum_{u \cong U} e(G(u, m))\right|=S^{\prime}(h), \quad \text { say, }
$$

where $G(u, m)=F(u, m)+\theta u, \theta$ is independent of $u$ and $m$, and $0 \leq \theta<1$. Let

$$
Q=\min \left(\sqrt[8]{h x M^{5} N^{-5}}, h x M^{-1} N^{-2}\right) x^{-\eta} .
$$

If $Q \leq 100$, then we get, trivially,

$$
\begin{aligned}
\left(\frac{v N^{2}}{h x}\right)^{1 / 2} S^{\prime}(h) & \ll\left(h x M N^{-1} Q^{-1}\right)^{1 / 2} \ll\left(M N^{1 / 2}+\sqrt[16]{(h x)^{7} M^{3} N^{-3}}\right) x^{\eta} \\
& \ll\left(v x^{-1 / 8}+\left(v^{5} x\right)^{1 / 8}\right) x^{10 \eta} \ll x^{1 / 2-10 \eta}
\end{aligned}
$$

If $Q>100$, by Cauchy's inequality, Lemma 1.3, and partial summation, we get, with some $Q_{1}, 1 \leq Q_{1} \leq Q$, the inequality

(7) $\left|S^{\prime}(h)\right|^{2} \ll(M U)^{2} Q^{-1}+M^{3 / 2} U Q^{-1}\left|\sum_{(u, q) \in D} \sum_{m \sim M} m^{-1 / 2} e(f(u, q, m))\right|$,

where $D=\left\{(u, q) \mid q \sim Q_{1}, u \cong U, u+q \cong U\right\}$ and $f(u, q, m)=$ $C_{3}\left(h x m^{-1}\right)^{1 / 2}\left(u^{1 / 2}-(u+q)^{1 / 2}\right)-q \theta$. We apply Lemma 1.4 to transform 
the sum over $m$ to a sum over $w$, where $w \cong W=N Q_{1} M^{-1}$. Then we change the order of summation, and estimate the sum over $w$ trivially, to get, with some $w$, the estimate

$$
\begin{aligned}
& M^{3 / 2} U Q^{-1}\left|\sum_{(u, q) \in D} \sum_{m \sim M} m^{-1 / 2} e(f(u, q, m))\right| \\
& \ll h x N^{-3 / 2} Q^{-1 / 2}\left|\sum_{(u, q) \in D_{1}} e(g(u, q))\right| \\
& \quad+(h x)^{2} Q^{-1 / 2} N^{-9 / 2}+(h x)^{2} Q^{-1} N^{-5}+(h x)^{2} M^{-1} N^{-4},
\end{aligned}
$$

where

$$
\begin{gathered}
D_{1}=D \cap\left\{(u, q)|1 \leq| C_{3}(2 w)^{-1}(h x)^{1 / 2} M^{-3 / 2}\left(u^{1 / 2}-(u+q)^{1 / 2}\right) \mid \leq \sqrt[3]{4}\right\} \\
g(u, q)=C_{4}\left(h x w\left(u^{1 / 2}-(u+q)^{1 / 2}\right)^{2}\right)^{1 / 3}-q \theta
\end{gathered}
$$

It is easy to verify that

$$
g(u, q) \widetilde{\Delta} C_{5}(h x w)^{1 / 3} u^{-1 / 3} q^{2 / 3}, \quad \Delta=Q_{1} U^{-1} .
$$

Choosing $X=U, Y=Q_{1}, F \cong N Q_{1}, \Delta=Q_{1} U^{-1}$ in Lemma 1.5, we find that

$$
\begin{aligned}
h x N^{-3 / 2} Q^{-1 / 2} & \left|\sum_{(u, q) \in D_{1}} e(g(u, q))\right| \\
\ll & \left(\sqrt[6]{(h x)^{9} Q^{2} M^{-3} N^{-13}}+\sqrt[6]{(h x)^{11} Q^{2} M^{-5} N^{-19}}\right. \\
& +\sqrt[10]{(h x)^{11} Q^{10} M^{-1} N^{-15}}+\sqrt[8]{(h x)^{15} Q^{3} M^{-7} N^{-27}} \\
& +\sqrt[4]{(h x)^{8} Q M^{-4} N^{-15}}+\sqrt[4]{(h x)^{6} Q^{3} M^{-2} N^{-10}} \\
& \left.+\sqrt{(h x)^{4} M^{-2} N^{-7}}\right) x^{2 \eta}
\end{aligned}
$$

From (6)-(9), we deduce that

$$
\text { (10) } \begin{aligned}
\left(\frac{v N^{2}}{h x}\right)^{1 / 2} S^{\prime}(h) \ll & \left(\sqrt[12]{(h x)^{3} M^{3} N^{5} Q^{2}}+\sqrt[12]{(h x)^{5} M N^{-1} Q^{2}}\right. \\
& +\sqrt[20]{(h x) M^{9} N^{15} Q^{10}}+\sqrt[16]{(h x)^{7} M N^{-3} Q^{3}} \\
& +\sqrt[8]{(h x)^{4} N^{-3} Q}+\sqrt[8]{(h x)^{2} v^{2} Q^{3}}+\sqrt[4]{(h x)^{2} N^{-1}} \\
& \left.+\sqrt[4]{(h x)^{2} M^{2} N^{-3} Q^{-1}}+\sqrt{h x M N^{-1} Q^{-1}}\right) x^{10 \eta}
\end{aligned}
$$

As we have already seen,

$$
\sqrt{h x M N^{-1} Q^{-1}} \ll x^{1 / 2-20 \eta},
$$

thus 


$$
\begin{aligned}
\sqrt[4]{(h x)^{2} M^{2} N^{-3} Q^{-1}} & \ll \sqrt[4]{(h x) M N^{-2} x} \ll \sqrt[8]{v^{4} x^{3} N^{-6}} \\
& \ll\left(v^{8} x^{3}\right)^{1 / 16} x^{10 \eta} \ll x^{1 / 2-20 \eta}
\end{aligned}
$$

From (9)-(11), we get

$$
\begin{aligned}
\left(\frac{v N^{2}}{h x}\right)^{1 / 2} S^{\prime}(h) \ll & \left(\sqrt[48]{(h x)^{13} M^{17} N^{15}}+\sqrt[48]{(h x)^{21} M^{9} N^{-9}}\right. \\
& +\sqrt[80]{(h x)^{9} M^{61} N^{35}}+\sqrt[128]{(h x)^{59} M^{23} N^{-39}} \\
& +\sqrt[64]{(h x)^{33} M^{5} N^{-29}}+\sqrt[64]{(h x)^{19} M^{31} N} \\
& \left.+\sqrt[4]{(h x)^{2} N^{-1}}\right) x^{10 \eta}+x x^{1 / 2-10 \eta} \\
\ll & \left(\sqrt[96]{v^{60} x^{13} N^{-4}}+\sqrt[96]{v^{60} x^{21} N^{-36}}+\sqrt[160]{v^{140} x^{9} N^{-52}}\right. \\
& +\sqrt[256]{v^{164} x^{59} N^{-124}}+\sqrt[128]{v^{76} x^{33} N^{-68}} \\
& \left.+\sqrt[128]{v^{100} x^{19} N^{-60}}+\sqrt[4]{v^{2} x N^{-1}}\right) x^{20 \eta}+x^{1 / 2-10 \eta} \\
\ll & \left(\sqrt[96]{v^{60} x^{12}}+\sqrt[160]{v^{140} x^{-4}}+\sqrt[128]{v^{82} x^{14}}+\sqrt[64]{v^{38} x^{8}}\right. \\
& \left.+\sqrt[64]{v^{50} x^{2}}+\sqrt[16]{v^{8} x^{3}}\right) x^{30 \eta}+x^{1 / 2-10 \eta} \ll x^{1 / 2-10 \eta}
\end{aligned}
$$

From (6), (7) and the above estimate, we get

$$
S(h) \ll\left(\left(\frac{v^{3}}{h x}\right)^{1 / 2}+\frac{v^{2}}{h x}+v x^{-1 / 4}\right) x^{20 \eta}+x^{1 / 2-8 \eta},
$$

hence

$$
\sum_{1 \leq h \leq H}|S(h)| \ll\left(v^{2} x^{-3 / 4}\right) x^{30 \eta}+v x^{-\eta} \ll v x^{-\eta} .
$$

The proof of Lemma 1 is finished.

Remark. The proof of Lemma 6 of [7] is false. On p. 191 we find the equality $E_{1}=E_{2}$, where

$$
\begin{aligned}
& E_{1}=\sum_{q=1}^{Q} \sum_{(h, m) \in \mathcal{P}_{q}} \sum_{\left(h^{\prime}, m^{\prime}\right) \in \mathcal{P}_{q}} \varepsilon_{h} \bar{\varepsilon}_{h^{\prime}} \sum_{n \sim N} e\left(\frac{x}{n}\left(\frac{h}{m}-\frac{h^{\prime}}{m^{\prime}}\right)\right), \\
& E_{2}=\sum_{h \sim J} \sum_{h^{\prime} \sim J} \varepsilon_{h} \bar{\varepsilon}_{h^{\prime}} \sum_{\substack{0 \leq k \leq M h h^{\prime}(J Q)^{-1} \\
0}} \sum_{\substack{m \sim M, m^{\prime} \sim M \\
m^{\prime} h-m h^{\prime}=k}} \sum_{n \sim N} e\left(\frac{k x}{m m^{\prime} n}\right),
\end{aligned}
$$

and

But we observe that

$$
\mathcal{P}_{q}=\left\{(h, m) \mid h \sim J, m \sim M, \frac{M(q-1)}{J Q} \leq \frac{m}{h} \leq \frac{M q}{J Q}\right\} .
$$

$$
0 \leq k \leq M h h^{\prime}(J Q)^{-1} \quad \text { and } \quad m^{\prime} h-m h^{\prime}=k
$$


cannot imply that there is a $q, 1 \leq q \leq Q$, such that both $(h, m) \in \mathcal{P}_{q}$ and $\left(h^{\prime}, m^{\prime}\right) \in \mathcal{P}_{q}$; this means that there is no one-to-one correspondence between the summation variables of $E_{1}$ and those of $E_{2}$, thus in general $E_{1} \neq E_{2}$.

2. Multiple exponential sums. Here we give several results on exponential sums, thus preparing an application of the sieve.

Lemma 2. For $v \cong M N, H=v x^{-1 / 2+\eta}, x^{0.6} \ll v x^{\varepsilon} \ll x^{2 / 3}$, $|a(n)|,|b(m)| \leq x^{\eta}$, and $v x^{-1 / 2+30 \eta} \ll N \ll\left(v x^{1 / 2}\right)^{1 / 7} x^{-30 \eta}$, we have

$$
\sum_{1 \leq h \leq H} \sum_{n \sim N} \sum_{m \sim M} a(n) b(m) e\left(\frac{h x}{m n}\right) \ll v x^{-\eta} .
$$

To prove Lemma 2, we need two lemmata.

Lemma 2.1. Let $H_{1} \geq H_{1}^{\prime} \geq 1, H_{2} \geq H_{2}^{\prime} \geq 1, n_{1}$ and $n_{2}$ be positive integers with $\left(n_{1}, n_{2}\right)=1$. Then

$$
\omega\left(n_{1}, n_{2} ; r\right):=\sum_{h_{1} n_{1}-h_{2} n_{2}=r}^{*} 1=\int_{0}^{1} \widehat{\omega}\left(n_{1}, n_{2} ; \theta\right) e(\theta r) d \theta
$$

and

$$
\int_{0}^{1}\left|\widehat{\omega}\left(n_{1}, n_{2} ; \theta\right)\right| d \theta \ll\left(1+H_{1} H_{2} n_{1}^{-1} n_{2}^{-1}\right)^{1 / 2}\left(\ln \left(2 H_{1} H_{2}\right)\right)^{2},
$$

where $*$ means the condition $H_{1}^{\prime} \leq h_{1} \leq H_{1}, H_{2}^{\prime} \leq h_{2} \leq H_{2}$.

Proof. This is Lemma 8 of [3].

Lemma 2.2. Suppose $\alpha=a / q+\theta / q^{2},(a, q)=1, q \geq 1,|\theta| \leq 1$. Then for any $\beta, U>0, P \geq 1$, we have

$$
\sum_{x=1}^{P} \min \left(U, \frac{1}{\|\alpha x+\beta\|}\right) \leq 6\left(\frac{P}{q}+1\right)(U+q \ln q) .
$$

Proof. This is Lemma 6 of Chapter 5 of [8].

Proof of Lemma 2. We assume that $x$ is irrational. Pick an integer $j$ such that $M \sim M_{1}=2^{j}$. We denote the triple exponential sum of Lemma 2 by $S(M, N)$. By Cauchy's inequality, we have $\left(m \simeq M_{1}\right.$ means $M_{1} \leq m$ $\left.\leq 4 M_{1}\right)$

$$
\begin{aligned}
x^{-2 \eta}|S(M, N)|^{2} & \ll M^{3 / 2} \sum_{m \simeq M_{1}} m^{-1 / 2}\left|\sum_{h=1}^{H} \sum_{n \sim N} a(n) e\left(\frac{h x}{m n}\right)\right|^{2} \\
& =M^{3 / 2} \sum_{h, h_{1}, n, n_{1}} a\left(n_{1}\right) \overline{a(n)} \sum_{m \simeq M_{1}} m^{-1 / 2} e\left(\left(\frac{h_{1}}{n_{1}}-\frac{h}{n}\right) \frac{x}{m}\right) .
\end{aligned}
$$


The terms with $h_{1} n=h n_{1}$ contribute at most $O\left(M^{2} H N x^{\eta}\right)$. We classify the remaining terms according to the value of $\left(n, n_{1}\right)$. After a familiar argument, we get

$$
\ll M^{3 / 2} \sum_{d \sim D} \sum_{\substack{n, n_{1} \sim N_{1} \\\left(n, n_{1}\right)=1}}\left|\sum_{r \sim R} \omega\left(n, n_{1} ; r\right) \sum_{m \simeq M_{1}} e\left(\frac{-r x}{d m n n_{1}}\right) m^{-1 / 2}\right|+M^{2} H N,
$$

for some $D, 1 \leq D \leq N, N_{1}=N / d$, and some $R, 1 \leq R \leq H N / D$. By Lemma 1.4, the innermost sum is equal to

$$
\begin{array}{rl}
\sum_{\alpha \leq u \leq \beta} u^{-1 / 2} & e\left(C_{6}\left(\frac{u r x}{d n n_{1}}\right)^{1 / 2}\right) C_{7} \\
& +O\left(M^{-1 / 2} \min \left(\left(\frac{M^{3} N^{2}}{R D x}\right)^{1 / 2}, \max \left(\frac{1}{\|\alpha\|}, \frac{1}{\|\beta\|}\right)\right)\right) \\
& +O\left(\frac{M^{3 / 2} N^{2}}{R D x} x^{\eta}\right)+O\left(M^{-1 / 2} x^{\eta}\right),
\end{array}
$$

where

$$
\alpha=\frac{r x}{\left(4 M_{1}\right)^{2} d n n_{1}}, \quad \beta=\frac{r x}{M_{1}^{2} d n n_{1}} .
$$

We consider the sum

$$
S^{*}(D, R)=M \sum_{d \sim D} \sum_{\substack{n, n_{1} \sim N_{1} \\\left(n, n_{1}\right)=1}} \sum_{r \sim R} \omega\left(n, n_{1} ; r\right) \min \left(\left(\frac{M^{3} N^{2}}{R D x}\right)^{1 / 2}, \frac{1}{\|\beta\|}\right) ;
$$

a similar sum with $\beta$ being replaced by $\alpha$ can be treated analogously.

We shall prove the following estimate for $S^{*}(D, R)$ :

$$
S^{*}(D, R) \ll v^{2} x^{-10 \eta} \quad \text { if } N \leq x^{1 / 4-20 \eta} .
$$

If $D \geq(M N)^{2} x^{-1-\eta}$, then we trivially get

$$
\begin{aligned}
S^{*}(D, R) & \ll M\left(\frac{M^{3} N^{2}}{R D x}\right)^{1 / 2} D \sum_{\substack{\left|n h_{1}-n_{1} h\right| \leq 2 R \\
h, h_{1} \leq H, n, n_{1} \leq 2 N / D}} 1 \\
& \ll(M N)^{5 / 2} H^{3 / 2} x^{-1 / 2} D^{-1} x^{\eta} \\
& \ll x^{1 / 2}(M N)^{1 / 2} H^{3 / 2} x^{2 \eta} \ll v^{2} x^{-1 / 4+20 \eta} .
\end{aligned}
$$

If $D \leq(M N)^{2} x^{-1-\eta}$, then we see that

$$
\frac{\beta}{r}=\frac{1}{q}+\frac{\theta}{q^{2}}, \quad \text { with } q=\left[d n n_{1} M_{1}^{2} x^{-1}\right] \geq 1 \text { and }|\theta| \leq 1,
$$


hence by Lemmata 2.1 and 2.2 , we have the estimate

$$
\begin{aligned}
S^{*}(D, R) & \ll M D \frac{N^{2}}{D^{2}}\left(1+\frac{D H}{N}\right) \max _{d, n, n_{1}} \sum_{r \sim R} \min \left(\left(\frac{M^{3} N^{2}}{R D x}\right)^{1 / 2}, \frac{1}{\|\beta\|}\right) \\
& \ll M N^{2}\left(\frac{R D x}{M^{2} N^{2}}+1\right)\left(\left(\frac{N^{2} M^{3}}{R D x}\right)^{1 / 2}+\frac{N^{2} M^{2}}{D x}\right) x^{\eta} \\
& \ll\left(v N x^{1 / 4}+v^{2} x^{-1 / 2} N^{2}+v^{5 / 2} N^{1 / 2} x^{-1 / 2}+v^{3} N x^{-1}\right) x^{10 \eta} \\
& \ll v^{2} x^{-10 \eta}
\end{aligned}
$$

provided only $N \leq x^{1 / 4-20 \eta}$. Thus anyhow (2.3) holds. By inserting (2.2) in (2.1), and taking into account (2.3), we get, after changing the order of summation, the following estimate (with $U=R D x v^{-2}$ ):

$$
\begin{aligned}
x^{-3 \eta}|S(M, N)|^{2} & \\
\ll & \frac{N M^{5 / 2}}{(R D x)^{1 / 2}} \sum_{d \sim D} \sum_{n, n_{1} \sim N_{1}} \sum_{u \cong U}\left|\sum_{r \in I} \omega\left(n, n_{1} ; r\right) e\left(C_{6}\left(\frac{u r x}{d n n_{1}}\right)^{1 / 2}\right)\right| \\
& \quad+v^{2} x^{-10 \eta},
\end{aligned}
$$

where $I$ is some subinterval of $[R, 2 R]$, which may depend on the variables outside the absolute value symbol. By Lemma 1.6 we get

$$
\begin{aligned}
& x^{-4 \eta}|S(M, N)|^{2} \\
\ll & \frac{N M^{5 / 2}}{(R D x)^{1 / 2}} \sum_{d \sim D} \sum_{n, n_{1} \sim N_{1}} \sum_{u \cong U}\left|\sum_{r \cong R} \omega\left(n, n_{1} ; r\right) e\left(C_{6}\left(\frac{u r x}{d n n_{1}}\right)^{1 / 2}+\theta r\right)\right| \\
& +v^{2} x^{-10 \eta}
\end{aligned}
$$

with some $\theta$, which is independent of the variables $r, u, n, n_{1}$ and $d$. Now we have arrived at (6.1) of [3], p. 325. The argument in what follows is exactly the same as in [3], and we get (cf. p. 329 of [3]), by the assumption of Lemma 2,

$$
\begin{aligned}
x^{-10 \eta}|S(M, N)|^{4} \ll & v^{2}\left(\left(\frac{H x}{v}\right)^{1 / 2} H^{2} N^{4}\right. \\
& +\left(\frac{H x}{v}\right) H^{3 / 2} N^{7 / 2}+\left(\frac{H x}{v}\right)^{1 / 2} N^{3} H^{2} M^{1 / 2} \\
& \left.+\left(\frac{H x}{v}\right) H^{4 / 3} M^{1 / 3} N^{8 / 3}\right)+\left(v^{2} x^{-10 \eta}\right)^{2} \\
& \ll v^{4} x^{-20 \eta} .
\end{aligned}
$$

Lemma 2 is proven. 
Lemma 3. For $v \cong M N, H=v x^{-1 / 2+\eta}, x^{0.6} \ll v x^{\varepsilon} \ll x^{3 / 4}$, $|a(n)|,|b(m)| \leq x^{\eta},(k, \lambda)$ an exponent pair, and

$$
v x^{-1 / 2+10 \eta} \leq N \leq \min \left(\left(v^{1-\lambda+k} x^{-k+\lambda / 2-1 / 4}\right)^{1 /(1-k+\lambda)}, x^{1 / 4}\right) x^{-20 \eta},
$$

we have

$$
\sum_{1 \leq h \leq H} \sum_{m \sim M} \sum_{n \sim N} a(n) b(m) e\left(\frac{h x}{m n}\right) \ll v x^{-\eta} .
$$

Proof. Note that (2.4) holds provided that

$$
v \leq x^{3 / 4-\varepsilon}, \quad N \leq x^{1 / 4-20 \eta} .
$$

In view of Lemma $2.1,(2.4)$ and $(2.5)$, we get

$$
x^{-4 \eta}|S(M, N)|^{2} \ll \frac{N^{3} M^{3 / 2}}{(R D)^{1 / 2}}\left|\sum_{r \sim R} e\left(F\left(r, d, n, n_{1}, u\right)\right)\right|+v^{2} x^{-10 \eta},
$$

where $F\left(r, d, n, n_{1}, u\right)=C_{6}\left(u r x /\left(d n n_{1}\right)\right)^{1 / 2}+\theta r$, for certain $d, n, n_{1}, u$ and $\theta$ with $|\theta| \leq 1$. It is easy to see that $F_{r}^{\prime}\left(r, d, n, n_{1}, u\right) \cong x D /(v N) \gg 1$, thus

$$
\sum_{r \sim R} e\left(F\left(r, d, n, n_{1}, u\right)\right) \ll R^{\lambda}\left(\frac{D x}{v N}\right)^{k} .
$$

Lemma 3 follows from (2.6), (2.7) and the fact that $R \ll H N$.

The last result of the section is

LEMmA 4. For $v \cong M N, H=v x^{-1 / 2+\eta}, x^{0.6} \ll v x^{\varepsilon} \ll x^{3 / 4},|a(n)| \leq x^{\eta}$ and $N \leq x^{3 / 8-\varepsilon}$, we have

$$
\sum_{1 \leq h \leq H} \sum_{m \sim M} \sum_{n \sim N} a(n) e\left(\frac{h x}{m n}\right) \ll v x^{-\eta} .
$$

To prove Lemma 4 we need again two lemmata.

Lemma 2.3. Let $\alpha, \beta, \gamma$ be real constants such that $(\alpha-1) \beta \gamma \neq 0$. Let $M, R, S, x \geq 1$ and let $\phi_{m}$ and $\psi_{r s}$ be complex numbers with modulus not exceeding 1 . Then

$$
\begin{aligned}
\sum_{m \sim M} \sum_{r \sim R} \sum_{s \sim S} \phi_{m} \psi_{r s} e & \left(\frac{x m^{\alpha} r^{\beta} s^{\gamma}}{M^{\alpha} R^{\beta} S^{\gamma}}\right) \\
\ll & \left(x^{1 / 4} M^{1 / 2}(R S)^{3 / 4}+M^{7 / 10} R S+M(R S)^{3 / 4}\right. \\
& \left.+x^{-1 / 4} M^{11 / 10} R S\right)(\ln (10 M R S))^{5}
\end{aligned}
$$

Proof. This is Theorem 3 of [3].

Lemma 2.4. Let $X \geq 100, Y \geq 100, A>0, f(x, y)=A x^{\alpha} y^{\beta}, F=$ $A X^{\alpha} Y^{\beta}, \alpha$ and $\beta$ being rational numbers (not positive integers). Suppose 
$F^{-2} X^{4} \leq Y^{3} N^{-\eta}$, and for $a_{x}$ and $b_{y}$ being complex numbers with modulus not exceeding 1 , define

$$
S(X, Y):=\sum_{(x, y) \in D} a_{x} b_{y} e(f(x, y)),
$$

where $D$ is some region contained in the rectangle $\{(x, y) \mid x \sim X, y \sim Y\}$ such that for a fixed $\widetilde{x}, \widetilde{x} \sim X$, the intersection $D \cap\{(\widetilde{x}, y) \mid y \sim Y\}$ has at most $O(1)$ segments. Then, for $W=X^{5}+Y^{5}$,

$$
\begin{aligned}
S(X, Y) \ll & \left(\sqrt[40]{F^{8} X^{24} Y^{27} W}+\sqrt[40]{F^{-4} X^{28} Y^{39} W}+\sqrt[4]{F^{-1} X^{3} Y^{5}}\right. \\
& +\sqrt[20]{F X^{13} Y^{19}}+\sqrt[4]{F X^{2} Y^{3}}+\sqrt[20]{F^{3} X^{14} Y^{7}} \\
& \left.+\sqrt[20]{F^{-3} X^{16} Y^{13}}+\sqrt[10]{F^{-3} X^{6} Y^{13}}\right)(F X Y)^{\eta}=: E
\end{aligned}
$$

Proof. Put $\delta=\eta^{2}$. By Lemma 1.6, we have (with $N=F X Y$ )

$$
N^{-\delta}|S(X, Y)| \ll \sum_{x \sim X}\left|\sum_{y \sim Y} C(y) e(f(x, y))\right|,
$$

where $C(y)=b_{y} e(\theta y)$ with some real number $\theta$ (which is independent of $x$ and $y)$. We choose

$$
Q=\left(F^{-1} X^{2} Y\right)^{2 / 5} \leq Y N^{-\delta} \quad \text { (by assumption). }
$$

If $Q \leq N^{\eta}$, then we trivially get

$$
|S(X, Y)| \ll N^{\eta / 2} X Y Q^{-1 / 2} \ll N^{\eta / 2}\left(\sqrt[5]{F X^{3} Y^{4}}\right) \ll E .
$$

Assume that $Q \geq N^{\eta}$. By Cauchy's inequality and Lemma 1.3, we get

$$
\begin{aligned}
N^{-3 \delta}|S(X, Y)|^{2} & \\
\ll & \frac{(X Y)^{2}}{Q}+\frac{X Y}{Q} \sum_{(y, q) \in D_{1}} \overline{C(y)} C(y+q) \sum_{x \sim X} e\left(A x^{\alpha} t(y, q)\right),
\end{aligned}
$$

where $D_{1}=\left\{(y, q) \mid y, y+q \sim Y, q \sim Q_{1}\right\}$ for some $Q_{1}, 1 \leq Q_{1} \leq Q$, and $t(y, q)=(y+q)^{\beta}-y^{\beta}$. Applying Lemma 1.4 to the innermost sum, we get

$$
\begin{aligned}
\sum_{x \sim X} e\left(A x^{\alpha} t\right) & \\
= & \sum_{u \in I} C_{7}\left|(A t)^{\gamma} u^{-1 / 2-\gamma}\right| e\left(C_{8}(A t)^{2 \gamma} u^{1-2 \gamma}\right) \\
& +O\left(\min \left(\left(\frac{X^{2} Y}{Q_{1} F}\right)^{1 / 2}, \frac{1}{\left\|g_{1}(y, q)\right\|}+\frac{1}{\left\|g_{2}(y, q)\right\|}\right)\right. \\
& \left.+\frac{X Y}{Q_{1} F}+\ln N+R(y, q)\right),
\end{aligned}
$$

where $I=\left(C_{9} A X^{\alpha-1}|t|, C_{10} A X^{\alpha-1}|t|\right), \gamma=1 /(2(1-\alpha)), g_{1}=\alpha A X^{\alpha-1} t$, $g_{2}=\alpha A(2 X)^{\alpha-1} t$, and $R(y, q)=0$ or $O\left(\left(X^{2} Y Q_{1}^{-1} F^{-1}\right)^{1 / 2}\right)$ according as 
none of the end points of $I$ is an integer or otherwise. For each fixed $q$, $q \sim Q_{1}$, we consider the sum

$$
\Phi=\sum_{y \sim Y} \min \left(\left(X^{2} Y Q_{1}^{-1} F^{-1}\right)^{1 / 2}, 1 /\|g(y)\|\right),
$$

where $g(y)$ is either $g_{1}(y, q)$ or $g_{2}(y, q)$. As both $g(y)$ and $g^{\prime}(y)$ are monotonic, and $g^{\prime}(y) \cong F Q_{1} X^{-1} Y^{-2}$, we can classify $y$ according to the integer part of $g(y)$, and it is easy to get

$$
\Phi \ll\left(1+F Q_{1} X^{-1} Y^{-1}\right)\left(\left(X^{2} Y Q_{1}^{-1} F^{-1}\right)^{1 / 2}+F^{-1} Q_{1}^{-1} X Y^{2} \ln N\right),
$$

which contributes to the RHS of (2.9) at most

$$
\begin{aligned}
& \ll \sqrt[10]{F^{-3} X^{16} Y^{13}}+\sqrt[5]{F^{-3} X^{6} Y^{13}}+\sqrt[10]{F^{3} X^{14} Y^{7}}+\sqrt{X Y^{2}} \\
& \ll E^{2} N^{-2 \eta}
\end{aligned}
$$

and similarly for the contribution of $R(y, q)$. From (2.9)-(2.11), after changing the order of summation, and estimating the sum over $u$ trivially, we get, with some $u,|u| \cong F Q_{1} X^{-1} Y^{-1}$, the estimate

$$
\begin{aligned}
& N^{-4 \delta}|S(X, Y)|^{2} \\
& \ll \frac{X Y}{Q}\left(1+\frac{F Q}{X Y}\right) A^{\gamma}\left(\frac{X Y}{F Q_{1}}\right)^{\gamma+1 / 2}\left|S_{1}\right|+N^{-\eta} E^{2}, \\
& S_{1}=S_{1}(u)=\sum_{(y, q) \in D_{2}} \overline{C(y)} C(y+q) t^{\gamma} e\left(C_{8}(A t)^{2 \gamma} u^{1-2 \gamma}\right), \\
& D_{2}=D_{1} \cap\left\{(y, q)\left|\left(C_{10} A X^{\alpha-1}\right)\right| t\left|\geq u \geq\left(C_{9} A X^{\alpha-1}\right)\right| t \mid\right\} .
\end{aligned}
$$

If $Q_{1} \leq N^{\eta}$, then we trivially have

$$
\begin{aligned}
\frac{X Y}{Q}\left(1+\frac{F Q}{X Y}\right) & A^{\gamma}\left(\frac{X Y}{F Q_{1}}\right)^{\gamma+1 / 2}\left|S_{1}\right| \\
& \ll \frac{X Y}{Q}\left(1+\frac{F Q}{X Y}\right) X Y^{3 / 2} F^{-1 / 2} N^{1 / 2} \\
& \ll \sqrt[10]{F^{-1} X^{12} Y^{21}}+\sqrt{F X^{2} Y^{3}} \\
& \ll \sqrt{F^{-1} X^{3} Y^{5}}+\sqrt[10]{F X^{13} Y^{19}}+\sqrt{F X^{2} Y^{3}} \ll N^{-\eta} E^{2} .
\end{aligned}
$$

Assume that $Q_{1} \geq N^{\eta}$. By Lemma 1.6 we get

$$
N^{-\delta}\left|S_{1}\right| \ll \sum_{y \sim Y}\left|\sum_{q \sim Q_{1}} C(y+q) e(\varphi q) t^{\gamma} e\left(C_{8}(A t)^{2 \gamma} u^{1-2 \gamma}\right)\right|,
$$

with some real number $\varphi$, independent of $y$ and $q$. Assume that $t(y, q)>0$ (otherwise we consider $-t$ ). Applying Cauchy's inequality and Lemma 1.3 
to (2.13), we get, with $Q_{2}=Q_{1}^{1 / 2} N^{-\delta}, G=\left(Q_{1} Y^{\beta-1}\right)^{\gamma}$,

$$
\begin{gathered}
N^{-3 \delta}\left|S_{1}\right|^{2} \ll \frac{\left(Y Q_{1} G\right)^{2}}{Q_{2}}+\frac{Y Q_{1}}{Q_{2}} \sum_{1 \leq\left|q_{1}\right| \leq Q_{2}}\left|S_{2}\left(q_{1}\right)\right|, \\
S_{2}\left(q_{1}\right)=\sum_{(y, q) \in D_{3}} \overline{C(y+q)} C\left(y+q+q_{1}\right) t_{1}\left(y, q, q_{1}\right) e\left(t_{2}\left(y, q, q_{1}\right)\right), \\
D_{3}=\left\{(y, q) \mid y \sim Y, q, q+q_{1} \sim Q_{1}\right\}, \\
t_{1}\left(y, q, q_{1}\right)=\left(t(y, q) t\left(y, q+q_{1}\right)\right)^{\gamma}, \\
t_{2}\left(y, q, q_{1}\right)=C_{8} u\left(\frac{A}{u}\right)^{2 \gamma}\left(t\left(y, q+q_{1}\right)^{2 \gamma}-t(y, q)^{2 \gamma}\right) .
\end{gathered}
$$

By writing $y+q=z$ we get

$$
\begin{gathered}
S_{2}\left(q_{1}\right)=\sum_{(z, q) \in D_{4}} \overline{C(z)} C\left(z+q_{1}\right) T_{1}\left(z, q, q_{1}\right) e\left(T_{2}\left(z, q, q_{1}\right)\right), \\
D_{4}=\left\{(z, q) \mid q, q+q_{1} \sim Q_{1}, z-q \sim Y\right\}, \\
T_{1}\left(z, q, q_{1}\right)=\left(t(z-q, q) t\left(z-q, q+q_{1}\right)\right)^{\gamma}, \\
T_{2}\left(z, q, q_{1}\right)=C_{8} u\left(\frac{A}{u}\right)^{2 \gamma}\left(t\left(z-q, q+q_{1}\right)^{2 \gamma}-t(z-q, q)^{2 \gamma}\right) .
\end{gathered}
$$

Again by Lemma 1.6, we get, with $I_{1}=[0.5 Y, 2.5 Y]$,

$$
N^{-\delta}\left|S_{2}\left(q_{1}\right)\right| \ll \sum_{z \in I_{1}}\left|\sum_{q \sim Q_{1}} T_{1}\left(z, q, q_{1}\right) e(\xi q) e\left(T_{2}\left(z, q, q_{1}\right)\right)\right|,
$$

with some real number $\xi$, independent of $z$ and $q$. Applying Cauchy's inequality and Weyl's lemma, we obtain, with $Q_{3}=Q_{1} N^{-\delta}$,

$$
\begin{aligned}
& N^{-3 \delta}\left|S_{2}\left(q_{1}\right)\right|^{2} \\
& \ll\left(Y Q_{1} G^{2}\right)^{2} Q_{3}^{-1}+Y Q_{1} Q_{3}^{-1} \sum_{1 \leq\left|q_{2}\right| \leq Q_{3}} \sum_{q \sim Q_{1}}\left|S_{3}\left(q, q_{1}, q_{2}\right)\right|, \\
& S_{3}\left(q, q_{1}, q_{2}\right)=\sum_{z \in I_{1}} T_{3}\left(z, q, q_{1}, q_{2}\right) e\left(T_{4}\left(z, q, q_{1}, q_{2}\right)\right), \\
& T_{3}\left(z, q, q_{1}, q_{2}\right)=T_{1}\left(z, q, q_{1}\right) T_{1}\left(z, q+q_{2}, q_{1}\right), \\
& T_{4}\left(z, q, q_{1}, q_{2}\right)=T_{2}\left(z, q+q_{2}, q_{1}\right)-T_{2}\left(z, q, q_{1}\right) .
\end{aligned}
$$

Let

$$
U(z)=T_{3}\left(z, q, q_{1}, q_{2}\right), \quad V(z)=T_{4}\left(z, q, q_{1}, q_{2}\right) .
$$

It is an easy exercise to verify that, for $z \in I_{1}$,

$$
\begin{gathered}
U(z) \cong G^{4}, \quad U^{\prime}(z) \cong G^{4} Y^{-1} \\
V(z) \cong\left|F q_{1} q_{2}\right| Y^{-1} Q_{1}^{-1}, \quad V^{\prime}(z) \cong \frac{F q_{1} q_{2}}{Y Q_{1} Y} .
\end{gathered}
$$


As $V(z)$ has nice properties with respect to the variable $z$, by partial summation and the exponent pair $(1 / 2,1 / 2)$, we obtain

(2.16) $S_{3}\left(q, q_{1}, q_{2}\right) \ll G^{4}\left(\left(\left|F q_{1} q_{2}\right| Y^{-2} Q_{1}^{-1}\right)^{1 / 2} Y^{1 / 2}+Y^{2} Q_{1}\left(\left|F q_{1} q_{2}\right|\right)^{-1}\right)$.

In view of (2.14) and (2.15), the first term in (2.16) contributes to (2.12) at most

$$
\begin{aligned}
& \ll N^{\eta / 2} \frac{X Y}{Q}\left(1+\frac{F Q}{X Y}\right) A^{\gamma}\left(\frac{X Y}{F Q_{1}}\right)^{1 / 2+\gamma} G \sqrt[16]{Y^{10} Q_{1}^{17} F^{2}} \\
& \ll\left(1+\frac{F Q}{X Y}\right) \sqrt[16]{F^{-6} X^{32} Y^{34} Q^{-7}} N^{\eta / 2} \\
& \ll\left(\sqrt[20]{F^{8} X^{24} Y^{27} W}+\sqrt[20]{F^{-4} X^{28} Y^{39} W}\right) N^{\eta / 2} \\
& \ll E^{2} N^{-\eta}
\end{aligned}
$$

and the second term in (2.16) contributes to (2.12) at most

$$
\begin{aligned}
& \ll N^{\eta / 2} \frac{X Y}{Q}\left(1+\frac{F Q}{X Y}\right) A^{\gamma}\left(\frac{X Y}{F Q_{1}}\right)^{1 / 2+\gamma} G\left(Q_{1}^{7} Y^{10} F^{-2}\right)^{1 / 8} \\
& \ll \sqrt[8]{Q^{-5} X^{16} Y^{22} F^{-6}}\left(1+\frac{F Q}{X Y}\right) N^{\eta / 2} \\
& \ll\left(\sqrt{F^{-1} X^{3} Y^{5}}+\sqrt[10]{F X^{13} Y^{19}}\right) N^{\eta / 2} \ll E^{2} N^{-\eta} .
\end{aligned}
$$

Finally, the term $\left(Y Q_{1} G^{2}\right)^{2} Q_{3}^{-1}$ together with the term $\left(Y Q_{1} G\right)^{2} Q_{2}^{-1}$ contributes to $(2.12)$ at most

$$
\begin{aligned}
& N^{\eta / 2} \frac{X Y}{Q}\left(1+\frac{F Q}{X Y}\right) A^{\gamma}\left(\frac{X Y}{F Q_{1}}\right)^{1 / 2+\gamma} Y G Q_{1}^{3 / 4} \\
& \ll X^{2} Y^{5 / 2} F^{-1 / 2} Q^{-3 / 4}\left(1+\frac{F Q}{X Y}\right) N^{\eta / 2} \ll E^{2} N^{-\eta} .
\end{aligned}
$$

The estimate (2.8) follows from the above observations.

Proof of Lemma 4. By Lemma 1.4, we obtain

$$
\begin{aligned}
\sum_{m \sim M} e\left(-\frac{h x}{m n}\right)= & \sum_{u \in I} C_{11}\left(n u^{-3} h^{-1}\right)^{1 / 4} e\left(C_{12}\left(\frac{h u x}{n}\right)^{1 / 2}\right) \\
& +O\left(\left(\frac{M^{3} N}{h x}\right)^{1 / 2}\right)+O\left(\frac{M^{2} N}{h x}\right)+O(\ln x),
\end{aligned}
$$

where $I=(\alpha, \beta), \alpha=h x /\left(4 M^{2} n\right), \beta=h x /\left(M^{2} n\right)$. We divide the sum in question into subsums of the form

$$
\sum_{h \sim H_{1}}\left|\sum_{n \sim N} \overline{a(n)} \sum_{m \sim M} e\left(-\frac{h x}{m n}\right)\right|=S\left(H_{1}\right), \quad \text { say },
$$


where $H_{1} \leq H$. By substituting (2.17) in (2.18), and exchanging the order of summation, we get, with an application of Lemma 1.6,

$$
S\left(H_{1}\right) \ll\left(\frac{M^{3} N}{H_{1} x}\right)^{1 / 2} \sum_{w \sim W}\left|\sum_{n \sim N} b(n) e(\theta n) e\left(C_{12}\left(\frac{w x}{n}\right)^{1 / 2}\right)\right| x^{\eta}+v x^{-10 \eta},
$$

where $W=H_{1}^{2} x v^{-1} M^{-1}$ and $\theta$ is some number (independent of the variables).

If $H_{1}^{8} \leq x^{\eta}\left(N x^{-5} v^{8}\right)$, then by Lemma 2.3 with $(r, s, m)=(1, w, n)$, we get the estimate

$$
\begin{aligned}
& x^{-3 \eta}\left|S\left(H_{1}\right)\right| \\
& \ll \sqrt[4]{H_{1}^{5} x^{2} N v^{-1}}+\sqrt[10]{H_{1}^{15} x^{5} N^{7} v^{-5}}+\sqrt[4]{H_{1}^{4} x N^{3}}+\sqrt[20]{H_{1}^{25} x^{5} N^{22} v^{-5}} \\
& \ll v\left(\sqrt[80]{N^{71} x^{-35}}+\sqrt[8]{N^{7} x^{-3}}+\sqrt[160]{N^{201} x^{-85}}+\sqrt[32]{N^{13} x^{-9}}\right)+v x^{-\varepsilon} \ll v x^{-\varepsilon} .
\end{aligned}
$$

If $H_{1}^{8}>x^{\eta}\left(N x^{-5} v^{8}\right)$, then we can apply Lemma 2.4 , with $(x, y)=(n, w)$, to get

$$
\begin{aligned}
x^{-3 \eta}\left|S\left(H_{1}\right)\right| \ll & \left(\frac{M^{3} N}{H_{1} x}\right)^{1 / 2}\left(\sqrt[40]{H_{1}^{62} x^{35} v^{-62} N^{56}}+\sqrt[40]{H_{1}^{72} x^{40} v^{-72} N^{56}}\right. \\
& +\sqrt[40]{H_{1}^{74} x^{35} v^{-74} N^{72}}+\sqrt[40]{H_{1}^{84} x^{40} v^{-84} N^{72}} \\
& +\sqrt[4]{H_{1}^{9} x^{4} v^{-9} N^{8}}+\sqrt[20]{H_{1}^{39} x^{20} v^{-39} N^{32}} \\
& +\sqrt[4]{H_{1}^{7} x^{4} v^{-7} N^{5}}+\sqrt[20]{H_{1}^{17} x^{10} v^{-17} N^{21}} \\
& \left.+\sqrt[20]{H_{1}^{23} x^{10} v^{-23} N^{29}}+\sqrt[10]{H_{1}^{23} x^{10} v^{-23} N^{19}}\right) \\
\ll & \sqrt[40]{H_{1}^{42} x^{15} v^{-2} N^{16}}+\sqrt[40]{H_{1}^{52} x^{20} v^{-12} N^{16}} \\
& +\sqrt[40]{H_{1}^{54} x^{15} v^{-14} N^{32}}+\sqrt[40]{H_{1}^{64} x^{20} v^{-24} N^{32}} \\
& +\sqrt[4]{H_{1}^{7} x^{2} v^{-3} N^{4}}+\sqrt[20]{H_{1}^{29} x^{10} v^{-9} N^{12}}+\sqrt[4]{H_{1}^{5} x^{2} v^{-1} N} \\
& +\sqrt[20]{H_{1}^{7} v^{13} N}+\sqrt[20]{H_{1}^{13} v^{7} N^{9}}+\sqrt[10]{H_{1}^{18} x^{5} v^{-8} N^{9}} \\
\ll & v x^{-10 \eta} .
\end{aligned}
$$

The proof of Lemma 4 is finished.

\section{Sieve methods}

3.1. Outline of setting. Let $v$ be a number such that

$$
x^{0.6-\varepsilon} \ll v \ll x^{0.723} \text {. }
$$


The sequence $\mathcal{A}$ is defined as

$$
\mathcal{A}=\left\{n \mid v \leq n \leq e v \text { and there exists an } m \text { with } x \leq m n \leq x+x^{1 / 2}\right\},
$$

where $e=2.71828 \ldots$ is the base of the natural logarithms. As usual, $S(\mathcal{A}, z)$ denotes the number of elements in $\mathcal{A}$ having no prime factors less than $z$. For $r$ a positive integer, let

$$
\left|\mathcal{A}_{r}\right|=\sum_{n \in \mathcal{A}, n \equiv 0(\bmod r)} 1 .
$$

It is easy to see that

$$
\begin{gathered}
\left|\mathcal{A}_{r}\right|=x^{1 / 2} / r+R(\mathcal{A}, r), \\
R(\mathcal{A}, r)=\sum_{v \leq r s \leq e v}\left(\psi\left(\frac{x+x^{1 / 2}}{r s}\right)-\psi\left(\frac{x}{r s}\right)\right)+O\left(\frac{x^{1 / 2}}{v}\right) .
\end{gathered}
$$

Let $V(z)=\prod_{p<z}(1-1 / p)$. With the above property of $\mathcal{A}$, we have

LEMMA 3.1.1. We have

$$
S(\mathcal{A}, z) \leq x^{1 / 2} V(z)\left(F\left(\frac{\ln D}{\ln z}\right)+O(\varepsilon)\right)+R^{+} \quad \text { if } 2 \leq z \leq D
$$

where

$$
R^{+}=\sum_{(D)} \sum_{\substack{r<D^{\eta} \\ r \mid P\left(D^{\eta}\right)}} C_{(D)}(r, \eta) \sum_{\substack{D_{i} \leq p_{i} \leq \min \left(z, D_{i}^{1+\eta}\right) \\ 1 \leq i \leq t}} R\left(\mathcal{A}, r p_{1} \ldots p_{t}\right),
$$

$t \gg 1, \sum_{(D)}$ is summation over all sequences $\left\{D_{i}\right\}_{i=1}^{t}$ with each $D_{i}$ of the form

such that

$$
D^{\eta^{2}\left(1+\eta^{2}\right)^{n}}, \quad n=0,1,2, \ldots
$$

$D_{1} \geq \ldots \geq D_{t} \geq D^{\eta^{2}}, \quad D_{1} \ldots D_{2 s} D_{2 s+1}^{3} \leq D \quad$ for all $0 \leq s \leq(t-1) / 2$,

and

$$
P\left(D^{\eta}\right)=\prod_{p<D^{\eta}} p, \quad\left|C_{(D)}(r, \eta)\right| \leq 1 .
$$

Also, for $p \sim \frac{1}{2} M, 2 \leq M \leq D^{1 / 2}$, we have

$$
S\left(\mathcal{A}_{p}, M\right) \geq \frac{x^{1 / 2}}{p} V(M)\left(f\left(\frac{\ln D}{\ln M}\right)+O(\varepsilon)\right)-\sum_{\substack{d<D \\ d \mid P(M)}} \lambda_{d} R(\mathcal{A}, p d)
$$

$\lambda_{d}$ being some numbers with $\left|\lambda_{d}\right| \leq D^{\eta}$. The functions $F$ and $f$ are the well-known functions in the linear sieve.

Proof. Both (3.1.1) and (3.1.2) come from [6]. 
We choose $P=v x^{-1 / 2+50 \eta}$, and

$$
\begin{aligned}
& x^{10 \varepsilon} D= \begin{cases}\left(x^{10} v^{-15}\right)^{1 / 2} & \text { for } x^{0.6} \ll v x^{\varepsilon} \leq x^{11 / 18}, \\
x^{3 / 8} & \text { for } x^{11 / 18}<v x^{\varepsilon} \leq x^{0.723+\varepsilon},\end{cases} \\
& x^{50 \eta} Q= \begin{cases}v^{-3} x^{2} & \text { for } x^{0.6} \ll v x^{\varepsilon} \leq x^{27 / 44}, \\
\left(v x^{1 / 2}\right)^{1 / 7} & \text { for } x^{27 / 44}<v x^{\varepsilon} \leq x^{67 / 104} \\
\left(v^{20} x^{-7}\right)^{1 / 36} & \text { for } x^{67 / 104}<v x^{\varepsilon} \leq x^{0.665}, \\
\left(v^{50} x^{-21}\right)^{1 / 70} & \text { for } x^{0.665}<v x^{\varepsilon} \leq x^{0.7} .\end{cases}
\end{aligned}
$$

LEMMA 5. We have

$$
S(\mathcal{A}, P) \leq x^{1 / 2} V(P)\left(F\left(\frac{\ln D}{\ln P}\right)+O(\varepsilon)\right) .
$$

Pr o of. By (3.1.1) it suffices to show that

$$
\begin{aligned}
\sum_{p_{1} \sim A_{1}} \ldots \sum_{p_{t} \sim A_{t}} \sum_{v^{\prime} \leq p_{1} \ldots p_{t} n \leq e v^{\prime}}\left(\psi\left(\frac{x+x^{1 / 2}}{r p_{1} \ldots p_{t} n}\right)-\psi\left(\frac{x}{r p_{1} \ldots p_{t} n}\right)\right) \\
\ll x^{1 / 2-0.75 \eta},
\end{aligned}
$$

where

$$
\begin{gathered}
A_{1} \gg A_{2} \gg \ldots \gg A_{t}, \\
A_{1} \ldots A_{2 s} A_{2 s+1}^{3} \leq D^{1+\eta} \quad \text { for } 0 \leq s \leq(t-1) / 2, \\
v^{\prime}=v r^{-1}, \quad r<D^{\eta} .
\end{gathered}
$$

Then, by a standard reduction, it is enough to establish

$$
\sum_{1 \leq h \leq H} \sum_{p_{1} \sim A_{1}} \ldots \sum_{p_{t} \sim A_{t}} \sum_{v \leq r p_{1} \ldots p_{t} n \leq e v} e\left(\frac{h x^{\prime}}{p_{1} \ldots p_{t} r n}\right) \ll v x^{-\eta},
$$

where $H=v x^{-1 / 2+\eta}, x \cong x^{\prime}$. Our aim is to arrange $\left\{r, p_{1}, \ldots, p_{t}, n\right\}$ into two subsets, so that we can produce from (3.1.3) an exponential sum of the type of Section 2, and then (3.1.3) will follow from the estimate given there.

We claim that either

$$
\begin{aligned}
& \text { there exists a subset } S \text { of }\{1,2, \ldots, t\} \text { with } \\
& \qquad P \leq \prod_{i \in S} A_{i} \leq Q x^{-2 \eta}:=Q_{1},
\end{aligned}
$$

or

$$
A_{1} \ldots A_{t} \leq x^{3 / 8-22 \eta}:=A_{0} .
$$

We assume the contrary, that is, neither (3.1.4) nor (3.1.5) is true, and deduce a contradiction. For $v \geq x^{11 / 18-\varepsilon}$, (3.1.5) is obvious. For $v<$ $x^{11 / 18-\varepsilon}$ we reason as follows. From $A_{1}^{3} \leq D^{1+\eta}<Q_{1}^{3}$, we have $A_{1}<P$. As $A_{0}<A_{1} \ldots A_{t} \ll P^{2} A_{3} \ldots A_{t}$, we have $A_{3} \ldots A_{t}>Q_{1}$. If $A_{3} A_{4}<P$, there 
must be a least $j$ such that $A_{3} A_{4} \ldots A_{j}>P$, hence $A_{3} A_{4} \ldots A_{j}>Q_{1}$; but then

$$
Q_{1}<A_{3} A_{4} \ldots A_{j} \ll\left(A_{3} \ldots A_{j-1}\right)\left(A_{3} A_{4}\right)^{1 / 2}<P^{3 / 2},
$$

a contradiction. If $A_{3} A_{4} \geq P$, then $A_{3} A_{4}>Q_{1}$, thus $A_{3}>Q_{1}^{1 / 2}$. But now

$$
Q_{1}^{5 / 2} \ll A_{1} A_{2} A_{3}^{3} \leq D^{1+\eta},
$$

also a contradiction. The proof of Lemma 5 is finished.

LEMma 6. We have

$$
\sum_{P \leq p<Q^{\prime}} S\left(\mathcal{A}_{p}, p\right) \geq \sum_{P \leq p<Q^{\prime \prime}} \frac{x^{1 / 2} V(p)}{p}\left(f\left(\frac{\ln (v / p)}{\ln p}\right)+O(\varepsilon)\right),
$$

where

$$
2 Q^{\prime \prime}=Q^{\prime}= \begin{cases}v^{10 / 37} & \text { for } x^{0.6} \ll v x^{\varepsilon} \leq x^{11 / 18} \\ Q & \text { for } x^{11 / 18}<v x^{\varepsilon} \leq x^{0.7}\end{cases}
$$

Proof. Let $J$ be the integer such that $1 \leq Q^{\prime} /\left(2^{J} P\right)<2$. Then

$$
\sum_{P \leq p<Q^{\prime}} S\left(\mathcal{A}_{p}, p\right) \geq \sum_{1 \leq j \leq J} \sum_{p \sim 2^{j-1} P} S\left(\mathcal{A}_{p}, 2^{j} P\right) .
$$

By (3.1.2), we have, with $D_{j}=\left(v /\left(2^{j} P\right)\right) x^{-\varepsilon}, P_{j}=2^{j} P$,

$$
S\left(\mathcal{A}_{p}, 2^{j} P\right) \geq \frac{x^{1 / 2}}{p} V\left(2^{j} P\right)\left(f\left(\frac{\ln D_{j}}{\ln P_{j}}\right)+O(\varepsilon)\right)-\sum_{d<D_{j}} \lambda_{d} R(\mathcal{A}, p d) .
$$

We now show that for each fixed $j, 1 \leq j \leq J$, we have

$$
\sum_{p \sim P_{j} / 2, d<D_{j}} \lambda_{d} R(\mathcal{A}, p d) \ll x^{1 / 2-\eta / 4} .
$$

Again, by the standard argument using the Fourier expansion of $\psi(\xi)$, to prove (3.1.8) it suffices to prove

$$
\sum_{1 \leq h \leq H} \sum_{p \sim P_{j} / 2} \sum_{d \leq D_{j}} \sum_{v \leq p d s \leq e v} e\left(\frac{h x^{\prime}}{p d s}\right) \ll v x^{-\eta},
$$

where $H=v x^{-1 / 2+\eta}, x \cong x^{\prime}$. At this stage the condition $v \leq p d s \leq e v$ can be removed by a familiar lemma (cf. [1]). Thus it is enough to get

$$
\sum_{1 \leq h \leq H} \sum_{n \sim N} \sum_{m \sim M} a(n) b(m) e\left(\frac{h \xi}{m n}\right) \ll v x^{-\eta},
$$

where $N=P_{j} / 2, M N \cong v, \xi \cong x,|a(n)| \leq x^{\eta},|b(m)| \leq x^{\eta}$. The above exponential sum is just of the form we considered in Section 2. As $P \ll$ 
$N=P_{j} / 2 \ll Q$ for $x^{0.6} \ll v x^{\varepsilon} \leq x^{0.7}$, we find that

(3.1.9) follows from

$$
\left\{\begin{array}{c}
\text { Lemma } 1.2 \text { when } x^{0.6} \ll v x^{\varepsilon} \leq x^{27 / 44} ; \\
\text { Lemma } 2 \text { when } x^{27 / 44}<v x^{\varepsilon} \leq x^{67 / 104} ; \\
\text { Lemma } 3 \text { with }(k, \lambda)=(2 / 7,4 / 7) \text { when } \\
\qquad x^{67 / 104}<v x^{\varepsilon} \leq x^{0.665} ; \\
\text { Lemma } 3 \text { with }(k, \lambda)=(11 / 30,16 / 30) \text { when } \\
\quad x^{0.665}<v x^{\varepsilon} \leq x^{0.7} .
\end{array}\right.
$$

Hence (3.1.8) is true. Using the asymptotic formula for $V(\cdot)$ and the well-known property of $f(\cdot)$, we have, for $p \sim P_{j} / 2=2^{j-1} P$,

$$
\begin{gathered}
V\left(2^{j} P\right)=V(p)\left(1+O\left(\frac{1}{\ln x}\right)\right), \\
f\left(\frac{\ln D_{j}}{\ln P_{j}}\right)=f\left(\frac{\ln (v / p)}{\ln p}\right)+O(\varepsilon) .
\end{gathered}
$$

Lemma 6 follows from (3.1.6)-(3.1.8) and (3.1.10).

3.2. The contribution of the range $(0.6-\varepsilon, 11 / 18-\varepsilon)$. We prove

Proposition 2. We have

$$
\sum_{21}:=\sum_{x^{0.6-\varepsilon} \leq p \leq x^{11 / 18-\varepsilon}} N(p) \ln p<0.02278 x^{1 / 2} \ln x .
$$

Unless otherwise specified, all symbols have the same meaning as in Subsection 3.1. Now, $v$ satisfies

$$
e^{-1} x^{0.6-\varepsilon} \leq v \leq x^{11 / 18-\varepsilon} .
$$

LEMMA 3.2.1.

$$
\sum_{\substack{p_{1} p_{2} \in \mathcal{A} \\ Q^{\prime} \leq p_{1} \leq \min \left(p_{2}, Q\right)}} 1=\frac{x^{1 / 2}}{\ln v}\left(\ln \left(\frac{5.4-8.1 s}{4 s-2}\right)+O(\varepsilon)\right),
$$

where $s=(\ln v) /(\ln x)$.

Proof. It is clear that

$$
\sum_{\substack{p_{1} p_{2} \in \mathcal{A} \\ Q^{\prime} \leq p_{1} \leq \min \left(p_{2}, Q\right)}} 1=\sum_{Q^{\prime} \leq p_{1} \leq Q} \sum_{v \leq p_{1} p_{2} \leq e v} \sum_{\substack{x \leq p_{1} p_{2} \\ m \leq x+x^{1 / 2}}} 1=U+V,
$$

where

$$
\begin{aligned}
U & =\sum_{Q^{\prime} \leq p_{1} \leq Q} \sum_{v \leq p_{1} p_{2} \leq e v} \frac{x^{1 / 2}}{p_{1} p_{2}}, \\
V & =\sum_{Q^{\prime} \leq p_{1} \leq Q} \sum_{v \leq p_{1} p_{2} \leq e v}\left(\psi\left(\frac{x+x^{1 / 2}}{p_{1} p_{2}}\right)-\psi\left(\frac{x}{p_{1} p_{2}}\right)\right) .
\end{aligned}
$$


By the Prime Number Theorem, we easily deduce that

$$
\begin{aligned}
U & =x^{1 / 2}\left(\sum_{Q^{\prime} \leq p_{1} \leq Q} \frac{1}{p_{1} \ln \left(v / p_{1}\right)}+O\left((\ln x)^{-2}\right)\right) \\
& =\frac{x^{1 / 2}}{\ln v}\left(\ln \left(\frac{5.4-8.1 s}{4 s-2}\right)+O(\varepsilon)\right)
\end{aligned}
$$

and the argument in the proof of Lemma 6 gives

$$
V=O\left(x^{1 / 2}(\ln x)^{-2}\right) .
$$

This proves Lemma 3.2.1.

Proof of Proposition 2. Let $A=0.6-\varepsilon, B=11 / 18-\varepsilon$, and $L=\ln x$. Then

$$
\sum_{21} \leq \sum_{A L-1 \leq k \leq B L}(k+1) \sum_{e^{k} \leq p \leq e^{k+1}} N(p) .
$$

For $v=e^{k}$, by Buchstab's identity it is easy to verify that

$$
\sum_{v \leq p \leq e v} N(p) \leq S(\mathcal{A}, P)-\sum_{P \leq p<Q^{\prime}} S\left(\mathcal{A}_{p}, p\right)-\sum_{\substack{p_{1} p_{2} \in \mathcal{A} \\ Q^{\prime} \leq p_{1} \leq \min \left(p_{2}, Q\right)}} 1,
$$

which, in conjunction with Lemmata 5,6 , and 3.2.1, leads to the estimate

$$
\sum_{21} \leq x^{1 / 2} L\left(I_{1}-I_{2}-I_{3}+O(\varepsilon)\right),
$$

where ( $\gamma$ being the Euler constant)

$$
\begin{gathered}
e^{\gamma} I_{1}=\int_{A}^{B} \frac{s}{s-1 / 2} F\left(\frac{10-15 s}{2 s-1}\right) d s \\
e^{\gamma} I_{2}=\int_{A}^{B} \int_{2.7}^{g(s)} f(t) d t d s, \quad g(s)=1 /(2 s-1), \\
I_{3}=\int_{A}^{B} \ln \left(\frac{5.4-8.1 s}{4 s-2}\right) d s .
\end{gathered}
$$

The following formulae are well known:

$$
\begin{gathered}
F(u)=\frac{2 e^{\gamma}}{u}(1+B(u)), \\
B(u)= \begin{cases}0 & \text { for } 0<u \leq 3, \\
\int_{2}^{u-1} \frac{\ln (t-1)}{t} d t & \text { for } 3 \leq u \leq 5 ;\end{cases} \\
f(u)=\frac{2 e^{\gamma} \ln (u-1)}{u} \quad \text { for } 2 \leq u \leq 4 ;
\end{gathered}
$$




$$
f(u)=\frac{2 e^{\gamma}}{u}\left(\ln (u-1)+\int_{3}^{u-1} \frac{d t}{t} \int_{2}^{t-1} \frac{\ln (s-1)}{s} d s\right)
$$

for $4 \leq u \leq 6$.

et $C=11 / 4$. By (3.2.1), after changing the order of integration, we find that

where

$$
I_{1}=I_{11}+I_{12}+I_{13}+I_{14}+O(\varepsilon)
$$

$$
\begin{aligned}
& I_{11}=\int_{A}^{B} \frac{4 u}{10-15 u} d u<0.02945 \\
& I_{12}=\left(\int_{2}^{C} \frac{\ln (t-1)}{t} d t\right)\left(\int_{A}^{B} \frac{4 u}{10-15 u} d u\right)<0.00273 \\
& I_{13}=\frac{4}{75}\left(\int_{C}^{4} \frac{(t-4) \ln (t-1)}{t(17+2 t)} d t\right)<-0.00042 \\
& I_{14}=\frac{40}{225}\left(\int_{C}^{4} \frac{\ln (t-1)}{t} \ln \left(\frac{17+2 t}{5+5 t}\right) d t\right)<0.00452
\end{aligned}
$$

thus

$$
I_{1}<0.03628 \text {. }
$$

By (3.2.2) and (3.2.3), after changing the order of integration, we deduce that

where

$$
I_{2}>I_{21}+I_{22}
$$

$$
\begin{aligned}
& I_{21}=\frac{1}{45}\left(\int_{2.7}^{4.5} \frac{\ln (t-1)}{t} d t\right)>0.01026 \\
& I_{22}=e^{-\gamma} f(4.5) \int_{4.5}^{5}\left(\frac{1}{2 t}-\frac{1}{10}\right) d t>0.00268 e^{-\gamma} f(4.5) .
\end{aligned}
$$

Since

$$
f(4.5)=\frac{2 e^{\gamma}}{4.5}\left(\ln 3.5+\int_{2}^{2.5} \frac{\ln (s-1)}{s} \ln \left(\frac{3.5}{s+1}\right) d s\right)>0.55788 e^{\gamma},
$$

we have

and thus

$$
I_{22}>0.00268 \cdot 0.55788>0.00149
$$

$$
I_{2}>0.01175
$$


Finally,

$$
I_{3}=\int_{A}^{B} \ln \left(\frac{5.4-8.1 s}{4 s-2}\right) d s>0.00175 .
$$

Combining (3.2.4), (3.2.5) and (3.2.6) proves Proposition 2.

3.3. The range $(11 / 18,27 / 44)$. For $11 / 18 \leq \alpha<\beta \leq 0.723$, let

$$
e^{\gamma} I(\alpha, \beta)=\int_{\alpha}^{\beta} \frac{t}{t-1 / 2} F\left(\frac{3}{8 t-4}\right) d t .
$$

Proposition 3. We have

$$
\begin{aligned}
\sum_{22}: & =\sum_{x^{11 / 18-\varepsilon} \leq p \leq x^{27 / 44-\varepsilon}} N(p) \ln p \\
& \leq\left(I\left(\frac{11}{18}, \frac{27}{44}\right)-0.00218\right) x^{1 / 2} \ln x .
\end{aligned}
$$

Proof. Let $A=11 / 18-\varepsilon, B=27 / 44-\varepsilon, L=\ln x$. Then

$$
\sum_{22} \leq \sum_{A L-1 \leq k \leq B L}(k+1) \sum_{e^{k} \leq p \leq e^{k+1}} N(p) .
$$

For $v=e^{k}$, Buchstab's identity gives

$$
\sum_{v \leq p \leq e v} N(p) \leq S(\mathcal{A}, P)-\sum_{P \leq p<Q^{\prime}} S\left(\mathcal{A}_{p}, p\right),
$$

which, in conjunction with Lemmata 5, 6 and the Prime Number Theorem, leads to the estimate

$$
\sum_{22} \leq x^{1 / 2} L\left(I_{1}-I_{2}+O(\varepsilon)\right)
$$

where

$$
\begin{aligned}
& e^{\gamma} I_{1}=\int_{A}^{B} \frac{t}{t-1 / 2} F\left(\frac{3}{8 t-4}\right) d t=\left(I\left(\frac{11}{18}, \frac{27}{44}\right)+O(\varepsilon)\right) e^{\gamma} \\
& e^{\gamma} I_{2}=\int_{A}^{B} \int_{G(s)}^{g(s)} f(t) d t d s, \quad g(s)=\frac{1}{2 s-1}, \quad G(s)=\frac{4 s-2}{2-3 s} .
\end{aligned}
$$

By (3.2.2) and (3.2.3), after changing the order of integration, we find that

$$
I_{2}>I_{21}+I_{22}+I_{23},
$$


where, with $C=8 / 3, D=20 / 7, E=22 / 5, F=4.5$,

$$
\begin{aligned}
& I_{21}=2 \int_{C}^{D} \frac{\ln (t-1)}{t}\left(\frac{2(1+t)}{4+3 t}-\frac{11}{18}\right) d t>0.0001, \\
& I_{22}=2 \int_{D}^{E} \frac{\ln (t-1)}{t}\left(\frac{27}{44}-\frac{11}{18}\right) d t>0.00202, \\
& I_{23}=2 \int_{E}^{F} \frac{\ln (t-1)}{t}\left(\frac{t+1}{2 t}-\frac{11}{18}\right) d t>0.00006 .
\end{aligned}
$$

Thus

$$
I_{2}>0.00218 \text {. }
$$

Proposition 3 is proved in view of (3.3.1) and (3.3.2).

3.4. The range $(27 / 44,67 / 104)$. We prove

Proposition 4. We have

$$
\begin{aligned}
\sum_{23}: & =\sum_{x^{27 / 44-\varepsilon} \leq p \leq x^{67 / 104-\varepsilon}} N(p) \ln p \\
& \leq\left(I\left(\frac{27}{44}, \frac{67}{104}\right)-0.01552\right) x^{1 / 2} \ln x .
\end{aligned}
$$

Pro of. Let $A=27 / 44-\varepsilon, B=67 / 104-\varepsilon, L=\ln x$. Then

$$
\sum_{23} \leq \sum_{A L-1 \leq k \leq B L}(k+1) \sum_{e^{k} \leq p \leq e^{k+1}} N(p) .
$$

For $v=e^{k}$, Buchstab's identity gives

$$
\sum_{v \leq p \leq e v} N(p)=\sum_{p \in \mathcal{A}} 1 \leq S(\mathcal{A}, P)-\sum_{P \leq p<Q^{\prime}} S\left(\mathcal{A}_{p}, p\right),
$$

which, in conjunction with Lemmata 5, 6 and the Prime Number Theorem, leads to the estimate

$$
\sum_{23} \leq x^{1 / 2} L\left(I_{1}-I_{2}+O(\varepsilon)\right)
$$

where

$$
\begin{aligned}
& e^{\gamma} I_{1}=\int_{A}^{B} \frac{t}{t-1 / 2} F\left(\frac{3}{8 t-4}\right) d t=e^{\gamma}\left(I\left(\frac{27}{44}, \frac{67}{104}\right)+O(\varepsilon)\right), \\
& e^{\gamma} I_{2}=\int_{A}^{B} \int_{G(s)}^{g(s)} f(t) d t d s, \quad g(s)=\frac{1}{2 s-1}, G(s)=\frac{12 s-1}{2 s+1} .
\end{aligned}
$$


By (3.2.2) and (3.2.3), after changing the order of integration, we find that

$$
I_{2}>I_{21}+I_{22}+I_{23}
$$

where, with $C=20 / 7, D=50 / 17, E=52 / 15, F=22 / 5$,

$$
\begin{aligned}
& I_{21}=\int_{C}^{D} \frac{2 \ln (t-1)}{t}\left(\frac{t+1}{12-2 t}-\frac{27}{44}\right) d t>0.00056 \\
& I_{22}=\int_{D}^{E} \frac{2 \ln (t-1)}{t}\left(\frac{67}{104}-\frac{27}{44}\right) d t>0.00789 \\
& I_{23}=\int_{E}^{F} \frac{2 \ln (t-1)}{t}\left(\frac{t+1}{2 t}-\frac{27}{44}\right) d t>0.00707 .
\end{aligned}
$$

Hence

$$
I_{2}>0.01552 .
$$

Proposition 4 follows from (3.4.1) and (3.4.2).

3.5. The range $(67 / 104,0.665)$. We prove

Proposition 5. We have

$$
\begin{aligned}
\sum_{24}: & =\sum_{x^{67 / 104-\varepsilon} \leq p \leq x^{0.665-\varepsilon}} N(p) \ln p \\
& <\left(I\left(\frac{67}{104}, 0.665\right)-0.00359\right) x^{1 / 2} \ln x .
\end{aligned}
$$

Proof. Let $A=67 / 104-\varepsilon, B=0.665-\varepsilon, L=\ln x$. Then

$$
\sum_{24} \leq \sum_{A L-1 \leq k \leq B L}(k+1) \sum_{e^{k} \leq p \leq e^{k+1}} N(p) .
$$

For $v=e^{k}$, by Buchstab's identity, we easily get

$$
\sum_{v \leq p \leq e v} N(p) \leq S(\mathcal{A}, P)-\sum_{P \leq p<Q^{\prime}} S\left(\mathcal{A}_{p}, p\right)
$$

which, in conjunction with Lemmata 5, 6 and the Prime Number Theorem, gives the estimate

$$
\sum_{24} \leq x^{1 / 2} L\left(I_{1}-I_{2}+O(\varepsilon)\right),
$$


where

$$
\begin{gathered}
e^{\gamma} I_{1}=\int_{A}^{B} \frac{t}{t-1 / 2} F\left(\frac{3}{8 t-4}\right) d t=\left(I\left(\frac{67}{104}, 0.665\right)+O(\varepsilon)\right) e^{\gamma}, \\
e^{\gamma} I_{2}=\int_{A}^{B} \int_{G(s)}^{g(s)} f(t) d t d s, \quad g(s)=\frac{1}{2 s-1}, G(s)=\frac{16 s+7}{20 s-7} .
\end{gathered}
$$

By (3.2.2) and (3.2.3), after changing the order of integration, we find that

$$
I_{2}=I_{21}+I_{22}+I_{23}+O(\varepsilon),
$$

where, with $C=14 / 5, D=50 / 17, E=100 / 33, F=52 / 15$,

$$
\begin{aligned}
& I_{21}=2 \int_{C}^{D} \frac{\ln (t-1)}{t}\left(0.665-\frac{7+7 t}{20 t-16}\right) d t>0.00065 \\
& I_{22}=2 \int_{D}^{E}\left(0.665-\frac{67}{104}\right) \frac{\ln (t-1)}{t} d t>0.00084 \\
& I_{23}=2 \int_{E}^{F} \frac{\ln (t-1)}{t}\left(\frac{1}{2}\left(1+\frac{1}{t}\right)-\frac{67}{104}\right) d t>0.0021 .
\end{aligned}
$$

Hence

$$
I_{2}>0.00359 .
$$

Proposition 5 is proved by virtue of (3.5.1) and (3.5.2).

3.6. The range $(0.665,0.7)$. We show

Proposition 6. We have

$$
\sum_{25}:=\sum_{x^{0.665-\varepsilon} \leq p \leq x^{0.7-\varepsilon}} N(p) \ln p \leq(I(0.665,0.7)-0.00149) x^{1 / 2} \ln x .
$$

Proof. Let $A=0.665-\varepsilon, B=0.7-\varepsilon, L=\ln x$. Then

$$
\sum_{25} \leq \sum_{A L-1 \leq k \leq B L}(k+1) \sum_{e^{k} \leq p \leq e^{k+1}} N(p) .
$$

For $v=e^{k}$, by Buchstab's identity, we deduce that

$$
\sum_{v \leq p \leq e v} N(p) \leq S(\mathcal{A}, P)-\sum_{P \leq p<Q^{\prime}} S\left(\mathcal{A}_{p}, p\right),
$$

which, in conjunction with Lemmata 5, 6 and the Prime Number Theorem, gives

$$
\sum_{25} \leq x^{1 / 2} L\left(I_{1}-I_{2}+O(\varepsilon)\right)
$$


where

$$
\begin{gathered}
e^{\gamma} I_{1}=\int_{A}^{B} \frac{t}{t-1 / 2} F\left(\frac{3}{8 t-4}\right) d t \\
e^{\gamma} I_{2}=\int_{A}^{B} \int_{G(s)}^{g(s)} f(t) d t d s, \quad g(s)=\frac{1}{2 s-1}, G(s)=\frac{20 s+21}{50 s-21} .
\end{gathered}
$$

By (3.2.2) and (3.2.3), after changing the order of integration, we get, with $C=2.5, D=2.8, E=100 / 33$,

$$
\begin{gathered}
I_{2}=I_{21}+I_{22}+O(\varepsilon), \\
I_{21}=2 \int_{C}^{D} \frac{\ln (t-1)}{t}\left(\frac{1}{2}\left(1+\frac{1}{t}\right)-\frac{21(1+t)}{50 t-20}\right) d t>0.00083, \\
I_{22}=2 \int_{D}^{E} \frac{\ln (t-1)}{t}\left(\frac{1}{2}\left(1+\frac{1}{t}\right)-0.665\right) d t>0.00066 .
\end{gathered}
$$

Thus

$$
I_{2}>0.00149 .
$$

By (3.6.1) and (3.6.2) we get the proposition.

3.7. The range $(0.7,0.723)$. We prove

Proposition 7. We have

$$
\sum_{26}:=\sum_{x^{0.7-\varepsilon} \leq p \leq x^{0.723}} N(p) \ln p<(I(0.7,0.723)+O(\varepsilon)) x^{1 / 2} \ln x .
$$

Proof. Let $A=0.7-\varepsilon, B=0.723, L=\ln x$. Then

$$
\sum_{26} \leq \sum_{A L-1 \leq k \leq B L}(k+1) \sum_{e^{k} \leq p \leq e^{k+1}} N(p) .
$$

For $v=e^{k}$, it is obvious that $\sum_{v \leq p \leq e v} N(p) \leq S(\mathcal{A}, P)$, which, in conjunction with Lemma 5 , gives

$$
\sum_{26} \leq x^{1 / 2} L\left(I_{1}+O(\varepsilon)\right),
$$

where

$$
e^{\gamma} I_{1}=\int_{A}^{B} \frac{t}{t-1 / 2} F\left(\frac{3}{8 t-4}\right) d t=(I(0.7,0.723)+O(\varepsilon)) e^{\gamma},
$$

which gives the proposition. 
Proof of Theorem. From Propositions 1 to 7, we get

$$
\sum_{p \leq x^{0.723}} N(p) \ln p \leq x^{1 / 2} \ln x(I(11 / 18,0.723)+0.6) .
$$

Let $A=11 / 18, B=0.723$ and $C=19 / 8$. By (3.2.1) we obtain

$$
\begin{aligned}
I(11 / 18,0.723) & =\frac{16}{3} \int_{A}^{B} t d t+\frac{8}{3} \int_{2}^{C} \frac{\ln (t-1)}{t}\left(\frac{(4 t+7)^{2}}{64(t+1)^{2}}-A^{2}\right) d t \\
& <0.39807+0.00043=0.3985,
\end{aligned}
$$

thus $I(11 / 18,0.723)+0.6<0.9985<1$, and our Theorem is proved in view of (1).

\section{References}

[1] R. C. Baker, The greatest prime factor of the integers in an interval, Acta Arith. 47 (1986), 193-231.

[2] E. Bombieri and H. Iwaniec, On the order of $\zeta\left(\frac{1}{2}+i t\right)$, Ann. Scuola Norm. Sup. Pisa 13 (1986), 449-472.

[3] E. Fouvry and H. Iwaniec, Exponential sums with monomials, J. Number Theory 33 (1989), 311-333.

[4] S. W. Graham, The greatest prime factor of the integers in an interval, J. London Math. Soc. 24 (1981), 427-440.

[5] D. R. Heath-Brown, Prime numbers in short intervals and a generalized Vaughan identity, Canad. J. Math. 34 (1982), 1365-1377.

[6] H. Iwaniec, A new form of the error term in the linear sieve, Acta Arith. 37 (1980), 307-320.

[7] C. H. Jia, The greatest prime factor of the integers in an interval (II), Acta Math. Sinica (Chinese) 32 (1989), 188-199. Cf. also doctoral thesis, Peking University, 1987.

[8] A. A. Karatsuba, Principles of Analytic Number Theory, Nauka, 1975 (in Russian).

[9] H. Liu, On square-full numbers in short intervals, Acta Math. Sinica (N.S.) 6 (1990), 148-164.

[10] S. H. Min, Methods in Number Theory, Vol. 2, Science Press, 1981 (in Chinese).

[11] K. Ramachandra, A note on numbers with a large prime factor, J. London Math. Soc. 1 (1969), 303-306.

[12] - , A note on numbers with a large prime factor (II), J. Indian Math. Soc. 34 (1970), 39-48.

206-10, BAO GUO STREET

HARBIN 150066, CHINA 